\title{
Performance Analysis of Next Generation Mobility Protocols for IMS/MMD Networks
}

\author{
Tsunehiko Chiba, Hidetoshi Yokota \\ KDDI R\&D Laboratories, Inc. \\ Saitama, Japan
}

\author{
Ashutosh Dutta, Dana Chee \\ Telcordia Technologies, Inc. \\ New Jersey, USA
}

\author{
Henning Schulzrinne \\ Columbia University, \\ New York, USA
}

\begin{abstract}
In an effort to provide seamless mobility support in IMS/MMD networks, operators need to choose a specific IPbased mobility protocol. However, there are several micro- and macro-mobility protocols available that the operators can choose from. Operators often face the challenges of selecting the appropriate mobility protocol that can provide the most cost efficient solution under a specific operating environment. Thus, it is important to analyze the effectiveness of these protocols before they are actually deployed in the IMS/MMD networks. In this paper, we analyze a number of candidate mobility protocols and conduct a performance analysis of some of these using a prototype implementation in an IPv6-based IMS/MMD testbed. These analyses provide us with some guidelines in terms of the applicability of these protocols when operators plan to deploy their IMS/MMD networks.
\end{abstract}

Keywords-component; Mobility Protocols, IMS/MMD, Handoff, Wireless Networks

\section{INTRODUCTION}

In recent years, various wireless network operators are planning to upgrade their cellular networks to IP-based networks so that they can offer flexible services to their customers. 3GPP (Third Generation Partnership Project) and 3GPP2 (Third Generation Partnership Project 2) have defined IMS (IP Multimedia Subsystem) [1] and MMD (MultiMedia Domain) [2] architecture, respectively, which can offer several key functions, such as session management, session mobility, quality of service and charging. SIP (Session Initiation Protocol) [3] has been adopted as the signaling protocol for session management, and DIAMETER [4] has been adopted to provide policy control and charging rules functionality in IMS/MMD architecture. On the other hand, several candidate IP-based mobility protocols that can provide seamless mobility features to the MNs (Mobile Nodes) are being considered. The IETF (Internet Engineering Task Force) has developed several IP-based mobility protocols which can be used as the candidate protocols to provide mobility support in an IMS/MMD network. Since each of these mobility protocols has its own pros and cons, it is important to evaluate them, carry out a comparative analysis and investigate its applicability to the evolving IMS/MMD network. In order to provide these services in a cost-effective manner, operators need to examine various issues associated with deployment, such as complexity, cost of operation and efficiency. The complexity and overhead of these issues depend upon a number of factors, such as signaling messages over the air, data overhead, and processing overhead at the end node that might affect the overall usage of the system's resources. These system resources could include different parameters, such as the MN's power, memory, network bandwidth etc. Wireless network operators want to reduce the complexity of network operations and provide services to users in the most cost-efficient manner. Thus, the choice of the mobility protocols is very important and should be considered while supporting the roaming users. Performance results from the prototype implementation of different types of mobility protocols, such as application layer mobility, hostcontrolled mobility and network-controlled mobility protocols over the IMS/MMD networks are useful for determining the level of complexity involved in each of these protocols.

The remainder of the paper is organized as follows. Section II describes some of the related work on performance analysis of the available mobility protocols. Section III introduces the candidate mobility protocols. Section IV explains how these protocols are implemented in an IMS/MMD network. Performance analyses for each of these mobility protocols under two different deployment scenarios are presented in Section V. Finally, Section VI concludes the paper.

\section{RERATED WORK}

There is a body of related work that compares mobility protocols at several different layers and evaluates their effectiveness. Akilyidz et al. [5] provide a taxonomy of the IPbased mobility protocols. Campbell et al. [6] provide a comparative analysis of micro-mobility protocols. Authors have cited a comparative analysis of application layer mobility and network layer mobility protocols in [7]. None of this research has studied the effect of IP-based mobility protocols on the performance of IMS/MMD networks. This paper analyzes some of the candidate mobility protocols in the context of IMS/MMD networks and provides a comparative analysis of some experimental results from the prototype implementation over two different kinds of access networks in a practical IMS/MMD testbed.

\section{MOBILITY PROTOCOLS}

A careful study of several cellular and IP-based mobility management techniques provides us with some insight into several of the basic functions which are needed during a mobility event. These functions can be categorized as network and resource discovery, link detection, configuration, authentication, binding update, and media redirection. Existing IP-based mobility management techniques can be implemented at any one of several layers of the protocol stack, such as the network layer, transport layer and application layer. MIPv4 (Mobile IPv4) [8] and MIPv6 (Mobile IPv6) [9] are the main 
network layer mobility protocols defined in the IETF. Cellular IP [10], HAWAII (Handoff Aware Wireless Access Internet Infrastructure) [11] and Proxy MIPv6 [12] are three of the micro-mobility protocols suitable for intra-domain mobility. The SIP-based mobility protocol [13] handles mobility by means of application layer signaling, such as SIP. HIP (Host Identity Protocol) [14] defines a new protocol layer between the network layer and the transport layer to provide terminal mobility in a way which is transparent to both of these layers.

We selected three candidate mobility protocols - MIPv6, Proxy MIPv6 and a SIP-based mobility protocol - for the analyses and performance evaluation under an IMS/MMD network. We briefly explain the operation of these three mobility protocols in the following subsections. A performance analysis and qualitative comparison can help operators to decide on the most appropriate set of protocols for their deployment.

\section{A. MIPv6}

Primarily, there are two mobile-controlled mobility protocols, MIPv4 and MIPv6, designed for IPv4 and IPv6 networks, respectively. MIPv4 introduces network elements, such as HA (Home Agent) and FA (Foreign Agent) and suffers from triangular routing and extra IP-IP encapsulation. Extra overhead and a longer traversal path cause performance degradation. MIPv6 resolved many of the deployment issues associated with MIPv4 and has been adopted as one of the candidate mobility protocols for the IMS/MMD networks. Thus, we focus our analysis on MIPv6. Since stateless address auto-configuration [15] is a standard part of MIPv6, MN always obtains a CoA (Care-of-Address) which is routable to a foreign network. Thus, there is no need to have an FA in the MIPv6 framework. When the MN moves to a new foreign network, it acquires a temporary CoA using stateless address auto-configuration or DHCPv6 [16]. However, MIPv6 needs to establish a tunnel between the MN and HA. Consequently, the overhead of tunneled packets will increase.

\section{B. Application Layer Mobility}

Mobility can also be handled using application layer signaling such as SIP. Application layer mobility eliminates the need for a mobility stack on the MN and does not need any other mobility elements, such as HA or FA, in the network. Thus, it facilitates easier deployment of mobility solutions for wireless networks. However, application layer mobility also has a number of shortcomings. It may take more time than network layer mobility because of application layer processing at the end nodes, it also requires a number of signaling handshakes and a longer traversal distance between the end nodes. In addition, it only supports mobility for SIP-based applications. If the application between the communicating hosts is SIP based, then mobility support can be provided using SIP signaling between the hosts. However, application layer mobility cannot be used to support any non-SIP-based application such as FTP and Telnet. Thus, if mobility support for all kinds of applications is needed, the application layer mobility protocol for SIP-based mobility may not be appropriate.

\section{Proxy MIPv6}

The advantage of local mobility management is to optimize many of the functions related to mobility and reduce the number of signaling messages over the air. A candidate mobility protocol such as Proxy MIPv6 is currently being discussed to support local mobility management. This protocol is designed to address local mobility and is controlled by the network elements in the edge routers. Proxy MIPv6 does not use any mobility stack on the $\mathrm{MN}$, but rather is assisted by the proxies on the edge routers to perform the mobility functions. These proxies are called MAG (Mobile Access Gateway) and co-locate with the edge routers. As long as the MN moves within the same domain which has MAGs, the MN assumes that it is in a home link. The MAG is responsible for sending the correct $\mathrm{MN}$ prefix as part of the router advertisement for stateless address auto-configuration. In general, a tunnel is not desirable on the MN because it adds extra processing and bandwidth constraints to the wireless hop. Thus, Proxy MIPv6 is preferred when mobility is confined within a domain and operators do not want to overload the MN's stack by setting up a tunnel between the MN and the HA. In the network localized mobility management paradigm, when the $\mathrm{MN}$ moves from one MAG to another MAG, and its movement is limited within one LMA (Local Mobility Anchor), the following operations must be performed. It can be broadly classified into a few steps such as layer 2 configuration, detection of a new link, router solicitation, access authentication, proxy binding update and address re-configuration.

\section{Analytical comparison}

In order to analyse the performance of the respective mobility protocols, it is important to compare how many mobility functions within a handoff event are managed by the respective candidate mobility protocols. Efficient operation of these functions will make the protocol more cost-effective in any deployment scenario. We select a few primitive functions and analyse how these protocols handle these functions.

1) Configuration: Configuration is one of the handoff processes when an $\mathrm{MN}$ is configured with a layer 3 identifier by obtaining an IP address from the server. A layer 3 address configuration consists of a few sub-processes, such as signaling exchanges between the client and the server, and actual address assignment. Both MIPv6 and SIP-based mobility management protocols share the common factor that each of these protocols does not require any foreign agent in the network. However, in the case of Proxy MIPv6, the MN does not need to go through the address configuration process when it moves within an LMA's domain.

2) Registration: In the case of MIPv6, the MN sends the binding update to the HA and $\mathrm{CN}$ (Corresponding Node). Thus, the binding update to the HA can be regarded as the registration process for MIPv6. In SIP-based mobility management, the MN updates its IP address with the visited SIP proxy or the home proxy. In the case of Proxy MIPv6, the MAG sends the binding update to the LMA. Thus, the LMA keeps track of the mobility proxy agent at which the $\mathrm{MN}$ is anchored. 
3) Tunneling: MIPv6 tunnels payload packets between the $\mathrm{MN}$ and the HA in both directions. In addition to the extra headers assigned to the original packet, additional encapsulation and de-capsulation processing is required on the $\mathrm{MN}$ and HA. Thus, each packet gets loaded with two extra headers, the first is the source address of the encapsulating agent and the second is the destination address, where the packet gets de-capsulated. In contrast, SIP-based mobility does not make use of tunneling as the media travels directly between the $\mathrm{MN}$ and $\mathrm{CN}$. Thus, processing delay due to encapsulation and tunneling overhead are avoided when SIPbased mobility is used. In the case of Proxy MIPv6, the tunnel is set up between the MAG and the LMA and tunneling overhead over the air is avoided.

4) Media redirection: Media redirection is a process of redirecting the media to the new location of the MN after the MN has moved to the new point-of-attachment. Delays in media redirection will depend upon the location of the anchor agent that actually redirects the media. Each of these mobility protocols adopts a different approach to reduce the delay due to media redirection. MIPv6 has adopted route optimization and hierarchical MIPv6 to reduce the traversal of media traffic to the new location. In the case of SIP-based mobility, media is transferred between the $\mathrm{MN}$ and $\mathrm{CN}$ directly without any media redirection path. Although Proxy MIPv6 is designed to support intra-domain mobility and thus is designed to reduce the delay due to media redirection, there are proposals to reduce such delay by adopting route optimization techniques [17].

5) QoS and Gate control: After all the handoff related operations are complete, a PCRF (Policy and Charging Rules Function) entity within an IMS/MMD network can control the media flow on the access gateway or HA/LMA. This operation affects the handoff delay and associated packet loss. In the IMS/MMD testbed, we have used DIAMETER as a protocol that can control the media traversal via the access router by installing a proper access list. Thus, handoff delay and packet loss during handoff vary depending upon whether or not the QoS and gate control mechanisms are in effect.

Table 1 shows an analytical comparison of how performance parameters are affected using these three mobility protocols. Based on the priority associated with these parameters, the operators can pick either of these protocols for deployment.

TABLE I. ANALYTICAL COMPALISON OF MOBILITY PROTOCOLS

\begin{tabular}{|l|l|l|l|l|}
\hline $\begin{array}{l}\text { Mobility } \\
\text { protocols }\end{array}$ & $\begin{array}{l}\text { Media path } \\
\text { distance }\end{array}$ & $\begin{array}{l}\text { Messages } \\
\text { over the air }\end{array}$ & $\begin{array}{l}\text { Tunnel } \\
\text { overhead }\end{array}$ & $\begin{array}{l}\text { End system } \\
\text { processing }\end{array}$ \\
\hline MIPv6 & Most & Medium & Most & Medium \\
\hline $\begin{array}{l}\text { Application } \\
\text { Layer }\end{array}$ & Least & Most & Least & Most \\
\hline $\begin{array}{l}\text { Proxy } \\
\text { MIPv6 }\end{array}$ & Medium & Least & Medium & Least \\
\hline
\end{tabular}

\section{IMPLEMENTATION OF MOBILITY PROTOCOLS IN IMS/MMD TESTBED}

Figure 1 shows an IPv6-based IMS/MMD multimedia testbed on which these three mobility protocols have been prototyped and Table 2 shows the specification of components used for implementation. This multimedia testbed is equipped with all the standard components, such as P-CSCF (Proxy-Call Session Control Function), S-CSCF (Serving-Call Session Control Function), HA/LMA, PCRF, DHCP server and MN. These components provide the required functionalities and demonstrate several basic features, such as call setup, call teardown, QoS control and roaming between the domains. The authors have provided functional details of the testbed in [7]. We have selected WiFi and emulated cdma2000 networks respectively as a representative case of $3 \mathrm{G}$ and 802.11 technologies. A WiFi network is populated with AR (Access Router) and a cdma2000 network is configured with emulated RAN (Radio Access Network) and PDSN (Packet Data Serving Node). We have implemented three types of mobility, MIPv6, SIP-based mobility and Proxy MIIPv6, in the testbed. Two types of handoff, handoff between ARs and handoff between PDSNs, can be carried out in the testbed. The PCRF functionality in the testbed helps to control media in the access gateway based on the QoS parameter of the existing session. We have carried out experiments to control the gate for media by interaction between PCRF and PDSN/AR, or between PCRF and HA/LMA during the handoff, and have measured the handoff delay. Analysis of experimental results for each of these mobility protocols under different access networks is provided in the next section.

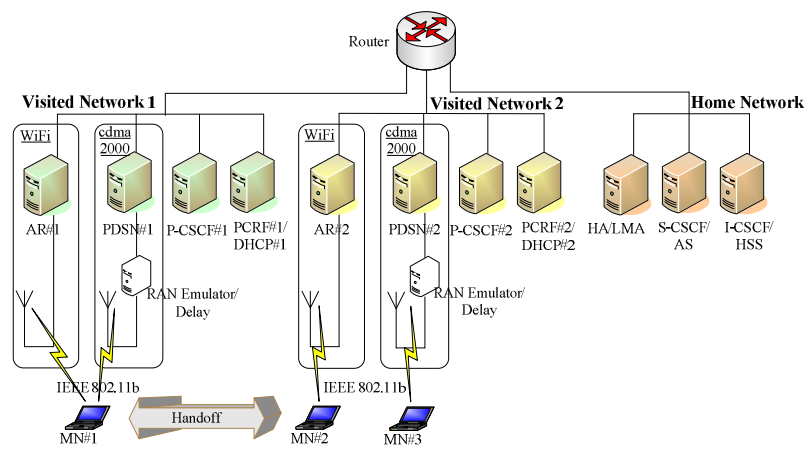

Figure 1. IMS/MMD multimedia testbed

TABLE II. SPECIFICATION OF COMPONENTS

\begin{tabular}{|l|l|}
\hline \multicolumn{2}{|l|}{ Server/Router } \\
\hline OS $^{\mathrm{a}}$ & Fedora Core 4 \\
\hline CPU & Pentium $42.8 \mathrm{GHz}$ \\
\hline Memory & $2 \mathrm{~GB}$ \\
\hline Network card & Intel PRO/1000MT \\
\hline Mobile Node & \multicolumn{2}{|l|}{} \\
\hline OS & Fedora Core 4 \\
\hline CPU & Pentium M $1.4 \mathrm{GHz}$ \\
\hline Memory & 1GB \\
\hline Network Card & IO-DATA WN-AG/CB2 (Mode: $802.11 \mathrm{~b}$ ) \\
\hline
\end{tabular}




\section{EXPERIMENTAL ANALYSIS OF MOBILITY PROTOCOLS}

In this section, we present the experimental results when these three different mobility protocols are used in an IMS/MMD testbed. We illustrate the call flows associated with the handoff operation for each of these protocols under two different access networks, WiFi and emulated cdma2000 which uses PPPoE (PPP over Ethernet) [18]. We analyze the experimental results associated with each of the functional components of the handoff operation.

\section{A. Handoff procedures for MIPv6}

In this subsection, we illustrate the call flows when MIPv6 is used as the mobility protocol for IMS/MMD networks.

1) Handoff in WiFi network: Figure 2 shows the handoff scenario when the $\mathrm{MN}$ moves from one AR to another $\mathrm{AR}$ in a WiFi network. MIPv6 is used to handle session continuity during this handover process. The $\mathrm{MN}$ sends a Router Solicitation (RS) message to the AR\#1 in order to obtain the foreign prefix which belongs to the AR\#1. The AR\#1 sends a Router Advertisement (RA) message to the MN including the foreign prefix. Since the AR\#1 assigns a unique foreign prefix to each $\mathrm{MN}$, the $\mathrm{MN}$ does not have to perform a DAD (Duplicate Address Detection) procedure. On receiving the foreign prefix, the $\mathrm{MN}$ sends a Binding Update message to the HA including CoA\#1 created through the stateless address auto-configuration procedure. In the IMS/MMD network, the $\mathrm{MN}$ has to register to the S-CSCF through the P-CSCF\#1 acquired by the $\mathrm{DHCP} \# 1$. When the $\mathrm{MN}$ requests a call to the $\mathrm{CN}$, the MN sends a SIP INVITE message to the $\mathrm{CN}$ through the P-CSCF\#1 and the S-CSCF. During the SIP INVITE procedure, the P-CSCF\#1 communicates with the PCRF\#1 in order to open the gate on the HA. After the gate is open, the $\mathrm{MN}$ and $\mathrm{CN}$ send and receive media data to each other.

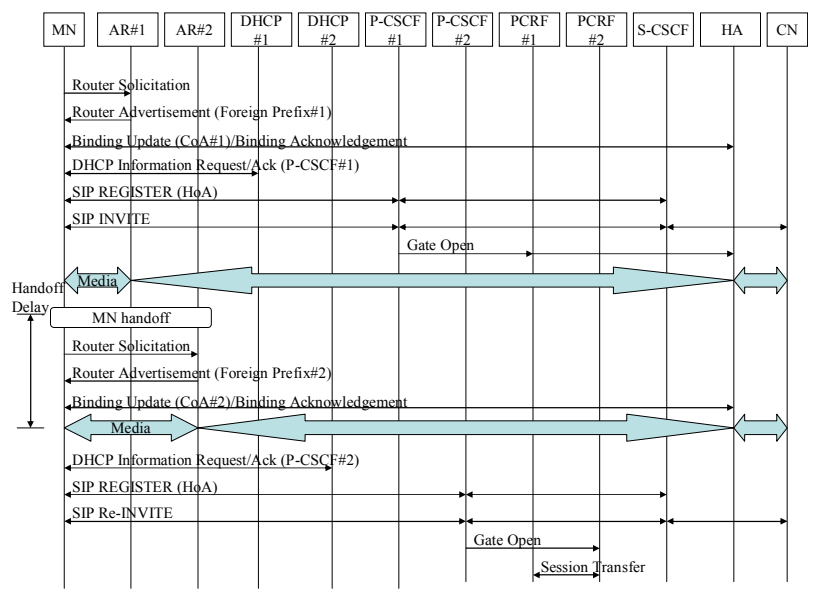

Figure 2. MIPv6 in WiFi network

When the MN moves to the AR\#2, the MN sends a Router Solicitation message to the AR\#2 and obtains the foreign prefix which belongs to the AR\#2. If the foreign prefix is different from the previous one, the $\mathrm{MN}$ sends a Binding Update message to the HA including CoA\#2. At this point, the
$\mathrm{MN}$ and the $\mathrm{CN}$ send and receive media data again. Consequently, the time of media packet loss is from when the $\mathrm{MN}$ discovers the new access point until the $\mathrm{MN}$ receives a Binding Acknowledge message from the HA. After receiving the Binding Acknowledge message, the MN performs DHCP, SIP registration and SIP re-INVITE procedures in order to update the SIP route information. During the re-INVITE procedure, the PCRF\#2 obtains the MN's session information from the PCRF\#1.

2) Handoff in emuloated cdma2000 network: Figure 3 shows the scenario when MIPv6 is applied to support handoff between the PDSNs in the emulated cdma2000 network. PPP setup consists of three negotiation procedures, the LCP (Link Control Protocol) phase, authentication phase and NCP (Network Control Protocol) phase. The establishment of a lower link in the cdma2000 network needs more time than the link setup in a regular WiFi network due to a lot of PPP negotiation messages. Besides the PPP configuration, the rest of the configuration mechanisms are similar to the $\mathrm{WiFi}$ network.

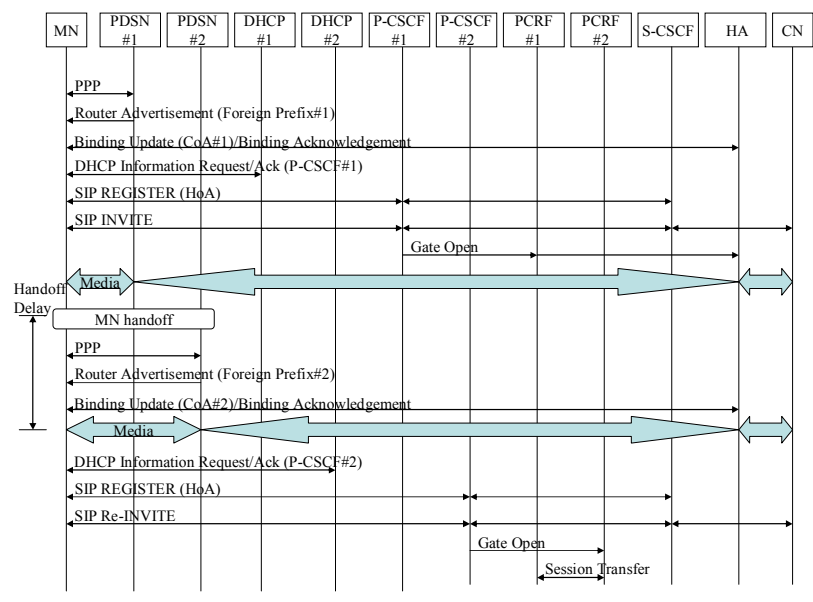

Figure 3. MIPv6 in emulated cdma2000 network

\section{B. Handoff procedures for SIP-based mobility}

In this subsection, we illustrate the call flows when a SIPbased mobility protocol is used for the mobility support in IMS/MMD networks.

1) Handoff in WiFi network: Figure 4 shows the call flow when SIP-based mobility is used in the WiFi network. Initially, the $\mathrm{MN}$ is in the current network anchored at AR\#1 and configures Address\#1 using assigned foreign prefix in the Router Advertisement message. Then, the $\mathrm{MN}$ performs DHCP, SIP registration and SIP INVITE procedures for setting up a call with the CN. During the SIP INVITE procedure, PCRF\#1 opens the gate on the AR\#1 based on the request from the $\mathrm{P}-\mathrm{CSCF} \# 1$. When the $\mathrm{MN}$ moves to the

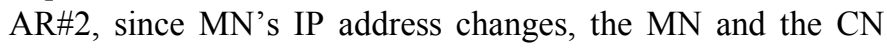
cannot send and receive media data until the SIP re-INVITE procedure and re-opening of the gate on the AR\#2 have been done. 


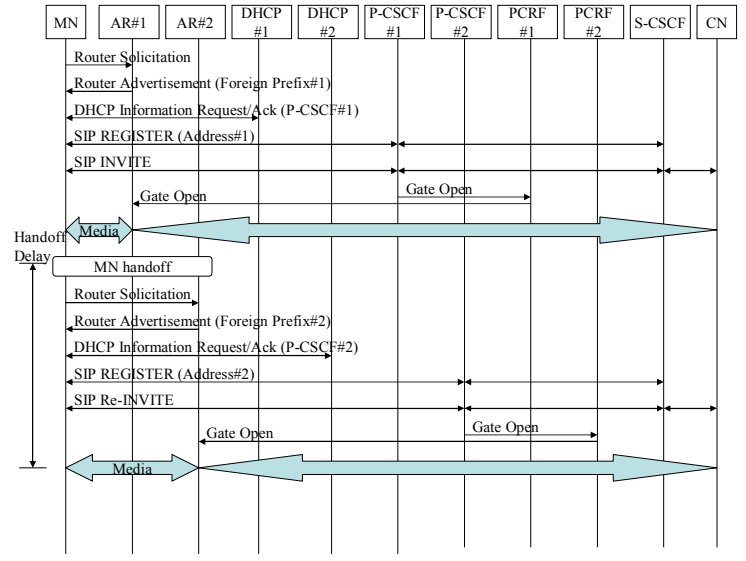

Figure 4. SIP-based mobility in WiFi network

2) Handoff in emulated cdma2000 network: Figure 5 shows the call flow when SIP-based mobility is used in an emulated cdma2000 network. The time of handoff delay in this scenario is the longest of all the scenarios cited in this paper due to the necessity for full SIP registration, re-INVITE procedures and a lot of PPP negotiation messages before opening the gate.

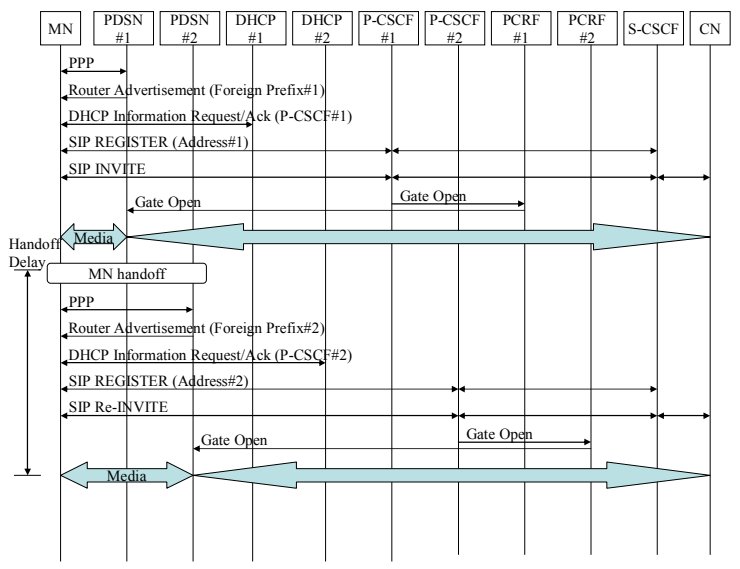

Figure 5. SIP-based mobility in emulated cdma2000 network

\section{Handoff procedures for Proxy MIPv6}

In the following subsections we describe the call flows of Proxy MIPv6 for IMS/MMD networks.

1) Handoff in WiFi network: Figure 6 shows the call flow when Proxy MIPv6 is used in the WiFi network. In the case of Proxy MIPv6, the AR\#1 sends a Proxy Binding Update (PBU) message to the LMA triggered by the received Router Solicitation from the MN. The LMA responds with a Proxy Binding Acknowledge (PBA) message including the MN's home prefix. Then, the AR\#1 includes the MN's home prefix in a Router Advertisement message. On receiving the Router Advertisement message, the MN creates the HoA through the stateless address auto-configuration mechanism and sends SIP registration to the S-CSCF through the P-CSCF\#1. When the
MN makes a call to the $\mathrm{CN}$, the MN sends a SIP INVITE message to the CN through the S-CSCF and the P-CSCF\#1. During the SIP INVITE procedure, the P-CSCF requests that the gate on the LMA be opened through the PCRF\#1. After the gate is opened, the $\mathrm{MN}$ and the $\mathrm{CN}$ can send and receive media data to and from each other. Compared to Binding Update messages in the MIPv6 case, Proxy Binding Update messages are not sent over the air. Consequently, Proxy MIPv6 is more efficient than MIPv6 in terms of conserving the wireless bandwidth. During the movement from the AR\#1 to the AR\#2, the AR\#2 sends a Proxy Binding Update message to the LMA so that the LMA can update the binding information of the MN. After receiving the Router Advertisement message from the AR\#2, the MN performs DHCP, SIP registration and SIP re-INVITE procedures in order to update the SIP route information. During the reINVITE procedure, the PCRF\#2 obtains the MN's session information from the PCRF\#1.

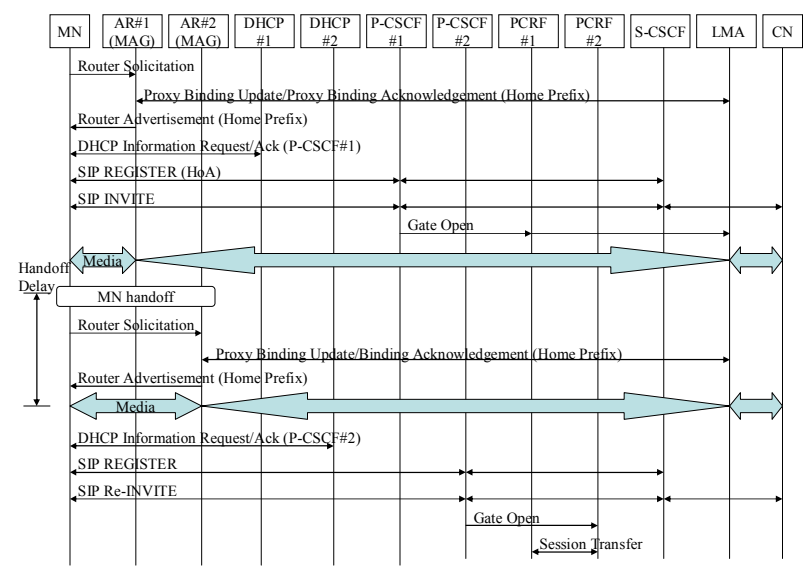

Figure 6. Proxy MIPv6 in WiFi network

2) Handoff in emulated cdma2000 network: Figure 7 shows the call flow when Proxy MIPv6 is used in an emulated cdma2000 network. Most of the handoff-related operations are similar to those for the WiFi network; however, it takes more time to set up the layer 2 link using PPP negotiation.

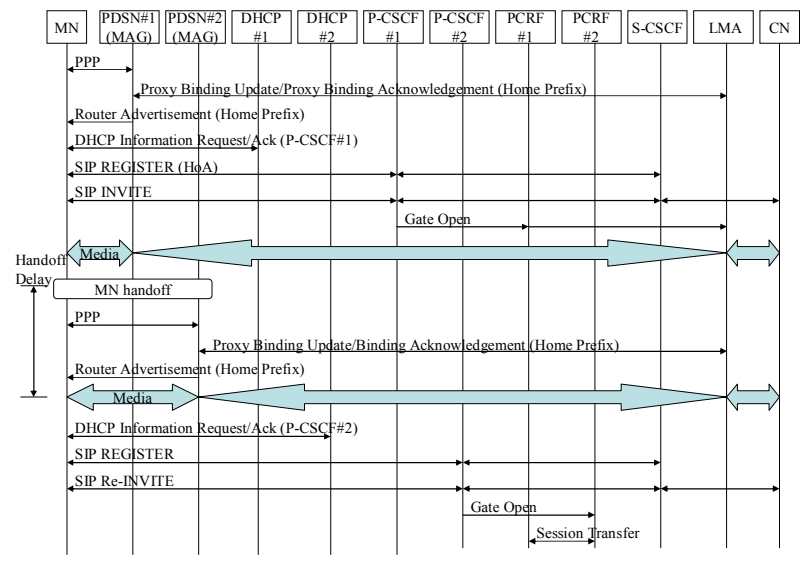

Figure 7. Proxy MIPv6 in emulated cdma2000 network 


\section{Handoff results}

Figure 8 shows the experimental results for the three mobility protocols for two kinds of access networks: WiFi network and emulated cdma2000 network with zero delay at the RAN emulator. We use the IEEE $802.11 \mathrm{~b}$ at the wireless part in both networks. The handoff time associated with different functional components is shown for each mobility protocol. In all cases, it takes about 2.5 seconds to establish an IEEE $802.11 \mathrm{~b}$ session during the handoff due to the wireless LAN association mechanism. Since this part is the same among three mobility protocols, we focus on the other handoff components. From Fig. 8, Proxy MIPv6 seems to be the most suitable candidate for both WiFi and emulated cdma2000 networks, as the $\mathrm{MN}$ spends the least amount of time for the layer 3 address configuration part when using Proxy MIPv6. The binding update in Proxy MIPv6 takes more time compared to the binding update in MIPv6 case due to the message conversion process associated with Proxy MIPv6, Router Solicitation and Router Advertisement at the AR or PDSN. SIP-based mobility takes the most time due to the dependence on SIP re-INVITE and SIP registration that help open the gate at the PDSN and AR.

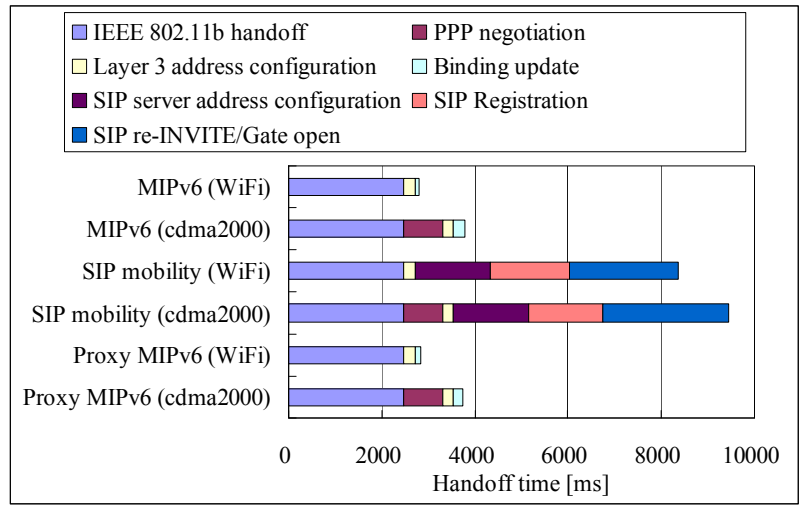

Figure 8. Comparison of functional delay components

Figure 9 shows total handoff time while increasing the access delay for the emulated cdma2000 RAN. RAN delay has less impact on Proxy MIPv6 compared to MIPv6 or SIP-based mobility because Proxy MIPv6 signaling messages are not exchanged over the air.

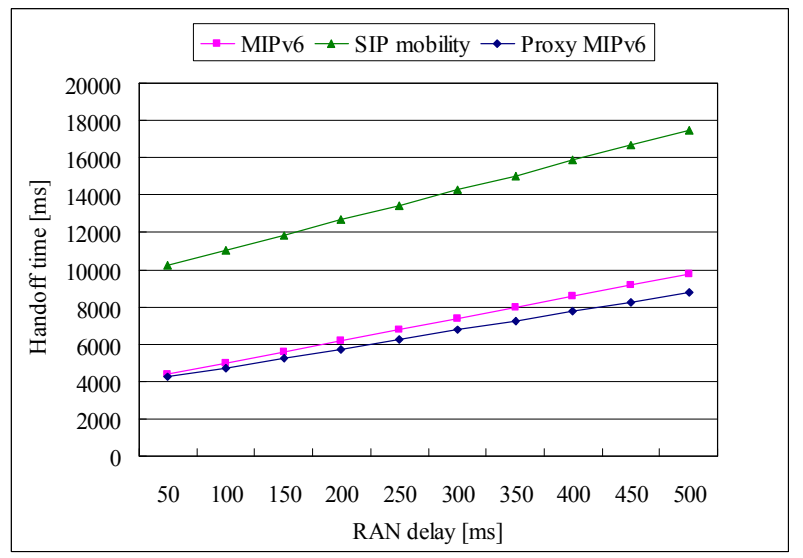

Figure 9. Handoff time with increasing RAN delay
In certain cases, such as in peer-to-peer applications, or in the case of an existing deployment where it is difficult to upgrade the routers, either SIP-based mobility or MIPv6 is preferred. Although SIP-based mobility helps to avoid the complexity associated with the additional home agent-related operations due to MIPv6 or upgrading the routers with MAG functionality, operators cannot adopt SIP-based mobility if the application is not SIP-based. On the other hand, if operators place special emphasis on handoff delay and mobility controlled by the network policy, Proxy MIPv6 is the most suitable mobility protocol.

\section{CONCLUSIONS}

In order to determine the suitability of three different mobility protocols for IMS/MMD networks, we have carried out analytical and performance analyses of these protocols in the IMS/MMD multimedia testbed. We have presented the experimental results using three mobility protocols over two different types of radio access networks in the testbed and evaluated the performance issues. These analyses and the experimental results provide certain guidelines regarding the type of mobility protocol that an operator should consider for deployment in the IMS/MMD network.

\section{REFERENCES}

[1] 3GPP, "IP Multimedia Subsystem (IMS); stage 2 (Release 7)" TS23.228 V7.10.0, Dec. 2007

[2] 3GPP2, "All-IP Core Network Multimedia Domain - IP Multimedia Subsystem Stage 2,” X.S0013-002-A, Nov. 2005

[3] J. Rosenberg et al., "SIP: Session Initiation Protocol," IETF RFC 3261, June 2002

[4] P. Calhoun et al., "Diameter Base Protocol," IETF RFC 3588, Sep. 2003

[5] I.F Akyildiz, J. Xie, S. Mohanty, "A survey of mobility management in next-generation all-IP-based wireless systems," IEEE Wireless Communications, vol 11, Aug. 2004

[6] A. Campbell, J. Gomez-Castellanos, "IP Micro-mobility protocols," vol 4, Oct. 2004

[7] A. Dutta et al., "Mobility testbed for 3GPP2-based Multimedia Domain Networks," IEEE Communication Magazine, vol 45, July 2007

[8] C. Perkins, "IP Mobility Support for IPv4," IETF RFC 3344, Jan. 2002

[9] D. Johnson et al., "Mobility Support in IPv6," IETF RFC 3775, June 2004

[10] L. Yi-Bing et al., "Mobility Management for Cellular Telephony Networks," IEEE Parallel \& Distributed Technology: Systems \& Technology, Dec. 1996

[11] Ramjee et al., "Hawaii: A Domain-based Approach for Supporting Mobility in Wide-area," ICNP'99, Nov. 1999

[12] S. Gundavelli et al., "Proxy Mobile IPv6," IETF draft-ietf-netlmmproxymip6-16, work in progress, May 2008

[13] E. Wedlund, H. Schulzrinne, "Mobility Support using SIP," ACM WoWMOM'99, 1999

[14] R. Moskowitz, P. Nikander, "Host Identity Protocol (HIP) Architecture," IETF RFC 4423, May 2006

[15] S. Thomson, T. Narten, "IPv6 Stateless Address Autoconfiguration," IETF RFC 2462, Dec. 1998

[16] R. Droms et al., "Dynamic Host Configuration Protocol for IPv6 (DHCPv6)," IETF RFC 3315, July 2003

[17] Park et al., "Localized Proxy-MIPv6 with Route Optimization in IPbased Networks," IEICE Transaction in Communication, E90-B No.12 pp.3682 - 3686, Dec. 2007

[18] L. Mamakos et al., "A Method for Transmitting PPP Over Ethernet (PPPoE),” IETF RFC 2516, Feb. 1999 